\title{
Study on Pore Distribution and Formation Rule of Sepiolite Mineral Nanomaterials
}

\author{
Qingguo Tang, ${ }^{1}$ Fei Wang, ${ }^{1,2}$ Mengran Tang, ${ }^{3}$ Jinsheng Liang, ${ }^{1}$ and Chongyan Ren ${ }^{1}$ \\ ${ }^{1}$ Institute of Power Source \& Ecomaterials Science, Hebei University of Technology, Tianjin 300130, China \\ ${ }^{2}$ Jiangsu Provincial Key Laboratory of Palygorskite Science and Applied Technology, Huaiyin Institute of Technology, \\ Huaian 223003, China \\ ${ }^{3}$ College of Engineering and Computing, Florida International University, Miami, FL 33174, USA
}

Correspondence should be addressed to Fei Wang, wangfei36777@163.com

Received 23 October 2012; Accepted 5 November 2012

Academic Editor: Su Chen

Copyright ( 2012 Qingguo Tang et al. This is an open access article distributed under the Creative Commons Attribution License, which permits unrestricted use, distribution, and reproduction in any medium, provided the original work is properly cited.

Theoretical research and specific surface area analysis of nitrogen adsorption indicated that a lot of structural micropores exist in sepiolite minerals fibers. However, the microporous size, existing form, and the distribution relationship between microporous structures were not proved yet. In this paper, the section TEM samples of nanofibers were prepared on the basis of the metal embedding and cutting technique, and the inner structure of sepiolite nanofibers was observed by TEM. The results showed that sepiolite fibers have multiplayer structure similar to concentric circles, and many micropores with the size of about 2-5 nm are normal and parallel to the $c$-axis. The reason for the previously mentioned phenomenon was explained by using BET analysis and $\mathrm{X}$-ray diffraction analysis results.

\section{Introduction}

Sepiolite is a kind of fibrous silicate clay mineral rich of magnesium with a structure of layer and chain. The study on structure of sepiolite could be dated back from 1930s, and Longchanbon studied the structure of sepiolite. Until 1955, Nagy and Bradley [1] proposed the first structural model and theoretical structure: $\mathrm{Si}_{12} \mathrm{Mg}_{9} \mathrm{O}_{30}(\mathrm{OH})_{6}\left(\mathrm{OH}_{2}\right)_{4} \cdot 6 \mathrm{H}_{2} \mathrm{O}$. Nagy believed that sepiolite crystal was amphibole chain composed of two pyroxene chains, with silicon-oxy tetrahedron on each side in standard spacing. Two layers of silicon-oxy tetrahedron parallel to $c 0$ axis had six silicon-oxy tetrahedron, separately. Structure units are connected to each other by four oxygen atoms on the apex angle, which formed channels with a sectional area of $0.38 \times 0.94 \mathrm{~nm}$ parallel to the chain.

Brauner and Preisinger [2] proposed the structural model of two amphibole chains composed by three pyroxene chains, as shown in Figure 1. According to Brauner's model, the theoretical structure of sepiolite was $\mathrm{Si}_{12} \mathrm{Mg}_{8} \mathrm{O}_{30}(\mathrm{OH})_{4}\left(\mathrm{OH}_{2}\right)_{4} \cdot 8 \mathrm{H}_{2} \mathrm{O}$, whose fibrous structures were composed of two layers of silicon-oxy tetrahedron units connected by oxygen atoms and central magnesium octahedron layers. Silicon-oxy tetrahedron layers were continuous, and were inverted at intervals of six tetrahedron units, leading to the existence of channels of dimensions of $0.37 \mathrm{~nm} \times 1.06 \mathrm{~nm}$ running parallel to the length of the fibers. The two models mentioned previously were not different obviously in terms of the sizes of crystal, but they had eight and nine of octahedron cations in Brauner's and Nagy's model, separately. Moreover, they had four hydroxyl groups, eight zeolite water, and sectional area of $0.3572 \mathrm{~nm}^{2}$ in Brauner's model; six hydroxyl groups, six zeolite water, and sectional area of $0.3816 \mathrm{~nm}^{2}$ in Nagy's model, separately. There were many reports about microstructure feature, crystal structure and thermal stability; however, the study on inner surface, adsorption, and channel structure of sepiolite was mainly by BET analysis [3-7]. The tested size and distribution of pores were different significantly from theoretical results due to the limitation of testing methods and accuracy [8-11].

In this paper, the inner structure of sepiolite nanofibers was observed by TEM in order to study the forms of existence 


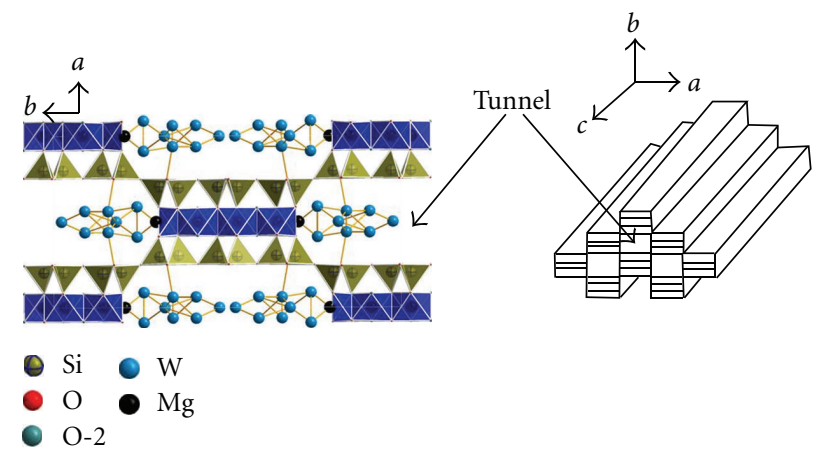

FIgURE 1: Crystal structure of sepiolite proposed by Brauner and Preisinger [2].

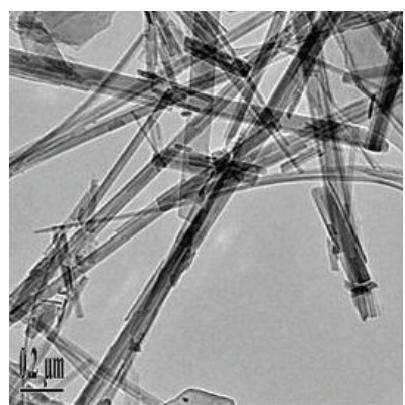

(a)

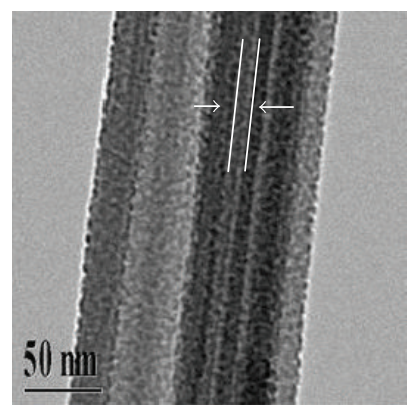

(b)

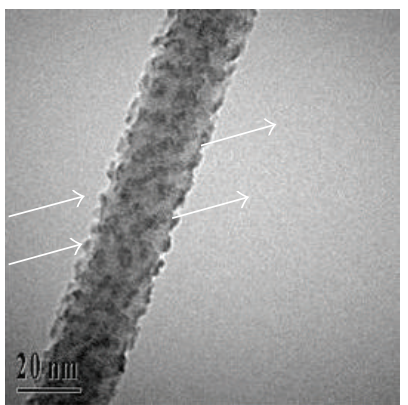

(c)

FIGURE 2: Microstructure of external surface of sepiolite samples.

and rules of distribution in sepiolite minerals fibers, which were further explained by using BET analysis and X-ray diffraction analysis results.

\section{Experimental Details}

Experimental details were as follows: hydrothermal sepiolite samples (Yi county, Hebei Province of China) were immersed in $0.1 \%$ of sodium pyrophosphate solution for $2 \mathrm{~h}$ in a reaction flask with stirring at a constant speed of $1500 \mathrm{rpm}$. Then the solutions were filtered and washed with distilled water until $\mathrm{pH}>6.5$. The samples were dried at $70^{\circ} \mathrm{C}$ for $8 \mathrm{~h}$ in the vacuum, and the purified samples were obtained. The process of TEM sample preparation is as follows [12]. A few sepiolite samples were placed on a polished metal strip, and the fine particles were encapsulated on the metal strip by the ion deposition technique. Then two surfaces of the encapsulated particle layer are polished until the fine particles are sufficiently exposed for the observation from both sides, and a micron-scaled thin film is obtained. Finally, the micron-scaled thin film is further reduced to a nanoscaled film penetrated by electron beams using ion beam thinner.

The microstructure of the samples was observed by scanning electron microscopy (Philips-XL30) at $25.0 \mathrm{kV}$ and $30 \mu \mathrm{A}$ and transmission electron microscopy (JEOL JEM2100). The X-ray diffraction (XRD) analysis was performed on a Philips-DMAX-2500 with $\mathrm{Cu} \mathrm{K} \alpha$ radiation. The specific surface area, pore volume, and average pore diameter of sepiolite are determined by the instrument of ASAP 2020.

\section{Results and Discussion}

3.1. Microstructure of Sepiolite Minerals. Figure 2 shows the TEM of the sepiolite samples. From Figure 2(a), it can be seen that sepiolite samples with diameter of $60-100 \mathrm{~nm}$ are fibrous minerals materials. From Figure 2(b), it can be seen that the prismatic sizes of sepiolite samples with the form of uneven surface multiprism vary from 10 to $40 \mathrm{~nm}$, and each surface of arris has enormous denticular microconvex without apparently regular distribution. From Figure 2(c), it can be seen that the prominent distance of microconvex mentioned previously with width of $2-5 \mathrm{~nm}$ and height of $1-2 \mathrm{~nm}$ is different, thus leading to many grooves with the tendency of spiral arrangement and distinct dislocation transform.

3.2. Specific Surface Area and Pore Character of Sepiolite Samples. The specific surface area of sepiolite samples is $160.77 \mathrm{~m}^{2} / \mathrm{g}$ with the $t$-Plot micropore area of $65.39 \mathrm{~m}^{2} / \mathrm{g}$ and $t$-Plot external surface area of $95.38 \mathrm{~m}^{2} / \mathrm{g}$. The adsorptive cumulative volume is $0.189 \mathrm{~cm}^{3} / \mathrm{g}$ within the range from 1.70 to $300.0 \mathrm{~nm}$, and the average pore diameter is $4.18445 \mathrm{~nm}$. The pore diameter distribution curves and isotherm linear pot are shown in Figures 3 and 4. From Figure 3, it can be seen that the $\mathrm{N}_{2}$ adsorption isotherm of sepiolite samples appearc hysteresis to a certain degree at $77 \mathrm{~K}$. From Figure 4, it can be seen that sepiolite fibers exist more mesopores with large pore volume. When $P / P_{0}=0-0.5$, they have larger adsorptive capacity, indicating that sepiolite 


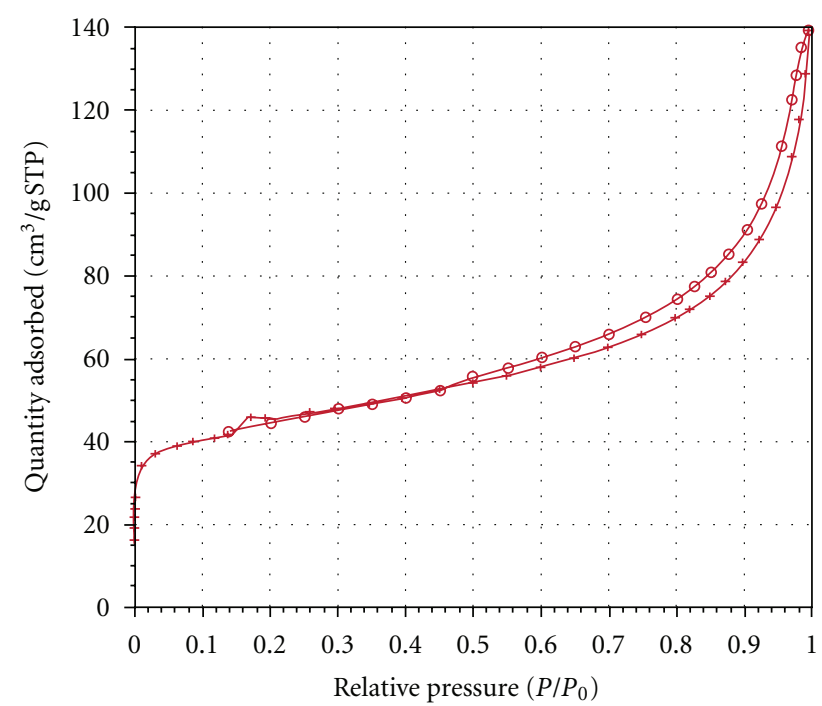

Figure 3: Absorption and desorption isotherm of sepiolite (“+” means absorption; “ $\bigcirc$ ” means desorption).

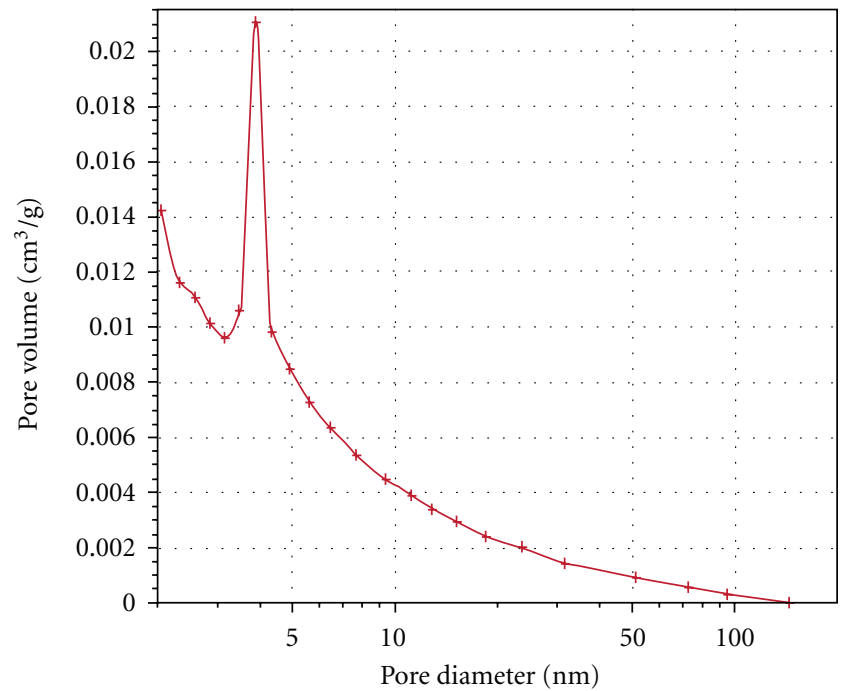

Figure 4: Pore diameter and pore volume distribution curve of sepiolite.

fibers contain considerable structural micropores with pore diameter $<1.7 \mathrm{~nm}$. The reason for the previously mentioned phenomenon is that adsorptive capacity increases along with the decrease of pore diameter, which proves the literature mentioned indirectly due to the limitation of BET method $[1,2]$. As shown in Figure 4, the pore diameter of sepiolite is mainly between $1.7 \sim 5 \mathrm{~nm}$, which proves that the adsorptive from is mainly mesopores adsorption. When $P / P_{0}=0.8 \sim 1$, the adsorption increases significantly, indicating the existence of micropores with diameter of 10-100 $\mathrm{nm}$. The results show that pore volume of sepiolite fibers with diameter of $3.7 \sim 4.1 \mathrm{~nm}$ is $11.2 \%$, and total area of sepiolite fibers with diameter of $<4.9 \mathrm{~nm}$ is $59.2 \%$, indicating that sepiolite fibers have part of larger channels.
3.3. Microstructure of the Section of Sepiolite Minerals. Figure 5 shows the section of sepiolite minerals. From Figure 5, it can be seen that part of dark area represents copper, and bright gray area represents the cross- and longitudinal-section of sepiolite fibers. Figure 5(b) is the partial enlarged detail of broken curve of Figure 5(a), which is likely to be a single fiber with diameter of $130 \mathrm{~nm}$ or multiple-strand fiber with diameter of 30-50 nm bundles. Many dark gray spots with diameter of 2-4 $\mathrm{nm}$ distribute regularly in the circle, which may be caused by the permeance of copper powders during the embedding. From Figure 5(a), it can be seen that gray area represents cross- and longitudinal-section of sepiolite fibers with irregular oval; the diameter of which is about $30-150 \mathrm{~nm}$. Due to the natural bend and difference of section degree of fibers, light area forms irregular alteration. Figure 5(d) is the partial enlarged detail of broken curve of Figure 5(c). From Figure 5(d), it can be seen the area pointed by arrow shows discontinuous states, indicating that fiber has at least more than four level alterations. Especially the oval area pointed by arrow four indicates that sepiolite fibers contain layer structure. The light and shade alteration of sepiolite section in the broken curve oval area in Figure 5(d) indicates the existence of considerable channels perpendicular to the inner surface of the fibers.

3.4. Microstructure Certification Inner Sepiolite Fibers. Figure 6 shows the longitudinal-section of a single fiber with diameter of $250 \mathrm{~nm}$ or two strings fibres. From the bright and dark difference in continuous line rectangle, it can be seen that many parallel arrangement and even breadth grooves or swells exist parallel to the $c$-axis. The width of grooves is about $2-5 \mathrm{~nm}$, and the smaller microswells (pointed by arrow) exists in larger grooves. The ribs of swells have many breakpoints, leading to the formation of channel. The ribs among breakpoint vary from 5 to $80 \mathrm{~nm}$; many of which are 


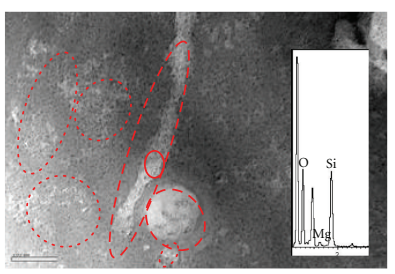

(a)

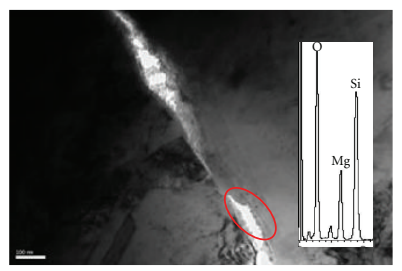

(c)

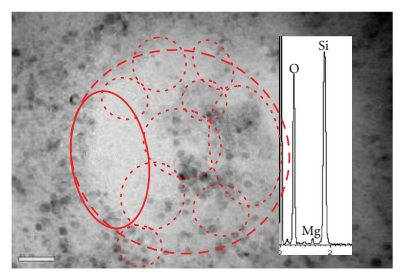

(b)

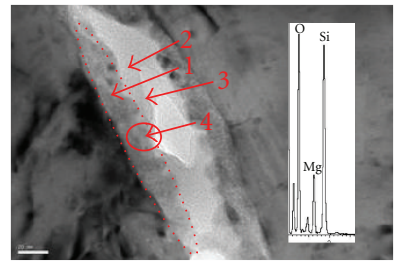

(d)
FIGURE 5: Microstructure of sepiolite section under HRTEM (a) cross and longitudinal section of sepiolite fibers; (b) cross section of sepiolite fiber bundles; (c) longitudinal section of sepiolite fibers; (d) partial enlarged detail of (c).

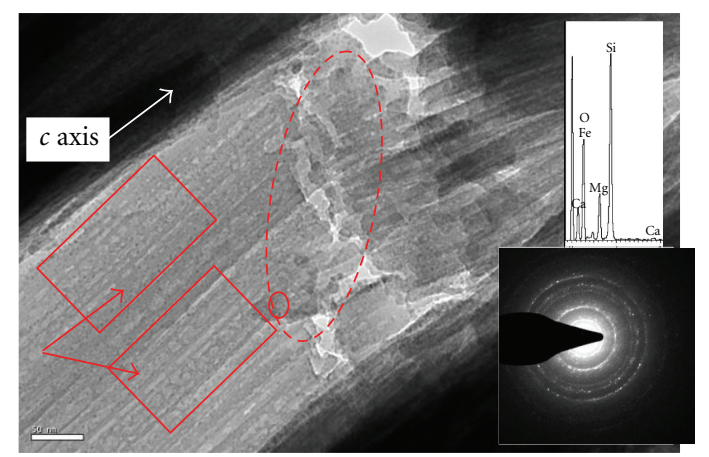

FIGURE 6: Diffraction spots and microstructure of sepiolite fibers.

10-25 nm. The diameter of channels parallel to the $c$-axis formed by breakpoints is mostly $3-5 \mathrm{~nm}(<10 \mathrm{~nm})$, and the ribs of bigger grooves or swells form many channels with the diameter of $2-4 \mathrm{~nm}$ (dark spots). The broken curve oval is the region of fiber breakdown, which shows many sub circular structural micropores with the diameter of $2-5 \mathrm{~nm}(<10 \mathrm{~nm})$. The diameters of micropores parallel to the $c$-axis are different significantly and are distributed irregularly, which in accordance with the results obtained by BET method. From diffraction spots in Figure 6, it can be seen that the diffraction spots are scattered and form obviously diffraction rings. The diffraction rings become wider caused by the irregularly distributed spots at the edge of inner diffraction rings, indicating the relative lower level of sepiolite minerals crystallization. And many breakpoints exist in diffraction rings, according to Bragg equation $2 d \sin \theta=n \lambda$, only if wavelength of X-ray is $n$ times as long as optical path difference between two crystal planes, diffraction appears. The existence of defect structure such as radial pores results in the discontinuation of crystal planes layered structure.

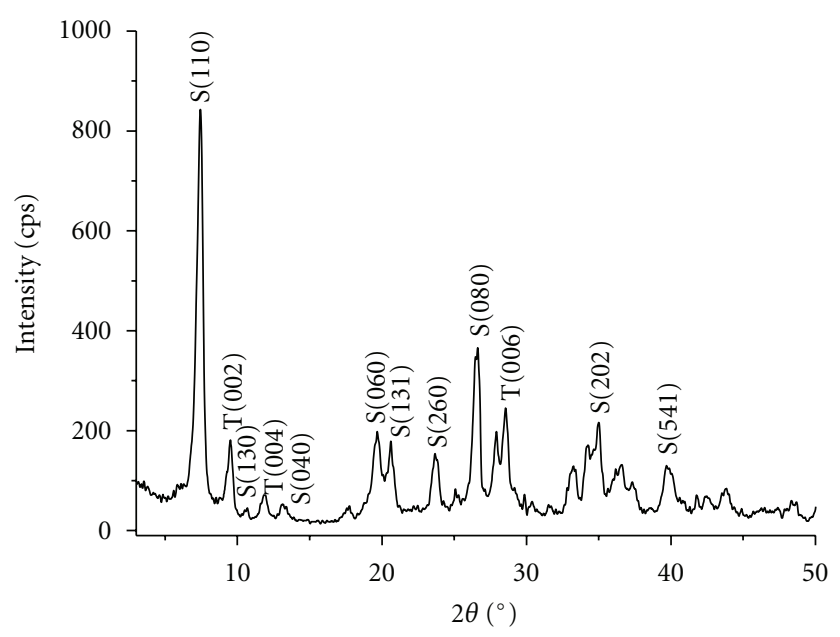

S: Sepiolite

T: Talc

FIGURE 7: XRD patterns of sepiolite minerals fibers.

3.5. X-Ray Diffraction Analysis of Sepiolite Fiber. In sepiolite diffraction spectrum, near $2 \theta=7.42^{\circ}$, a strong diffraction peak appears (as shown in Figure 7), which is characteristic of layered two-dimensional lattice structure minerals, proving that sepiolite is a kind of layered fibrous structure mineral similar to concentric circles. According to Brauner's model and its theoretical crystal planes spacing should be about $1.26 \mathrm{~nm}$, almost similar to the value tested by XRD $(121 \mathrm{~nm})$. The intensity of the diffraction peak of sepiolite crystal planes [110] is twice as high as other crystal planes, indicating that crystallization degree of sepiolite crystal planes [110] is significantly higher than that of other crystal planes, which becomes the foundation of sepiolite mineral crystal. Other crystals have many defects due to low crystallization degree, leading to uneven surfaces of fibers.

3.6. Reasons for Fibrous Structure, Uneven Surface, and Formation of Channels of Sepiolite. The interlayer spacing of sepiolite crystal planes is about $1.24 \mathrm{~nm}$; in it there exist a lot of pore channels in different sizes perpendicular to the $c$-axis, and its pore channel area is about $10-16 \mathrm{~nm}^{2}$ with $75 \mathrm{~nm}^{2}$ as the largest [3], which is in accordance with results of the observation basically. In sepiolite mineral, silica tetrahedron net surfaces form layered crystal with half of magnesium oxygen octahedron through regular reversal, producing many longitudinal channels parallel to the $c$-axis, as shown in Figure 8.

From the view of Brauner's model, the lower density areas of atom appear in the reverse parts of silica tetrahedron nets surface, which is perhaps the key to forming a lot of channels perpendicular to the fiber axis. Although BET method is unlikely to detect related information of micropores with diameter smaller than $1.7 \mathrm{~nm}$ quantitatively, the observation in TEM can see a certain number of subprime structural micropores with the diameter less than $2.0 \mathrm{~nm}$ in structure layer. The XRD analysis shows that the interplanar distance of sepiolite mineral is only $1.2 \mathrm{~nm}$ and the width 


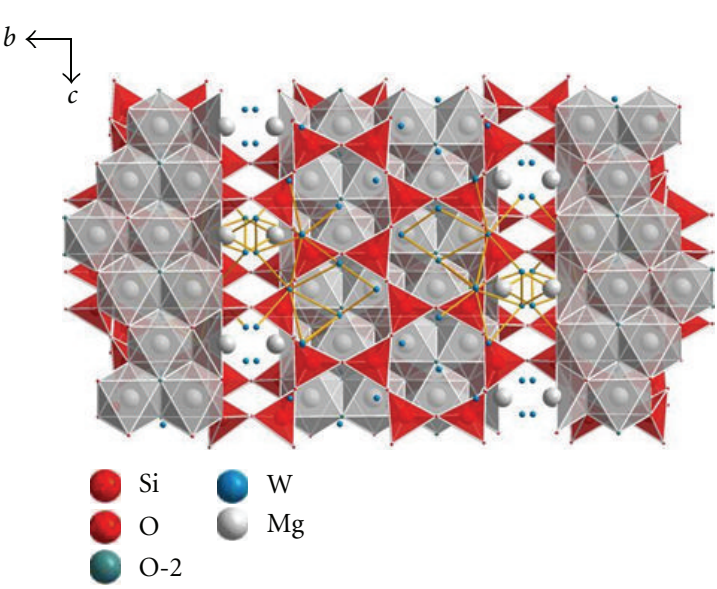

(a)

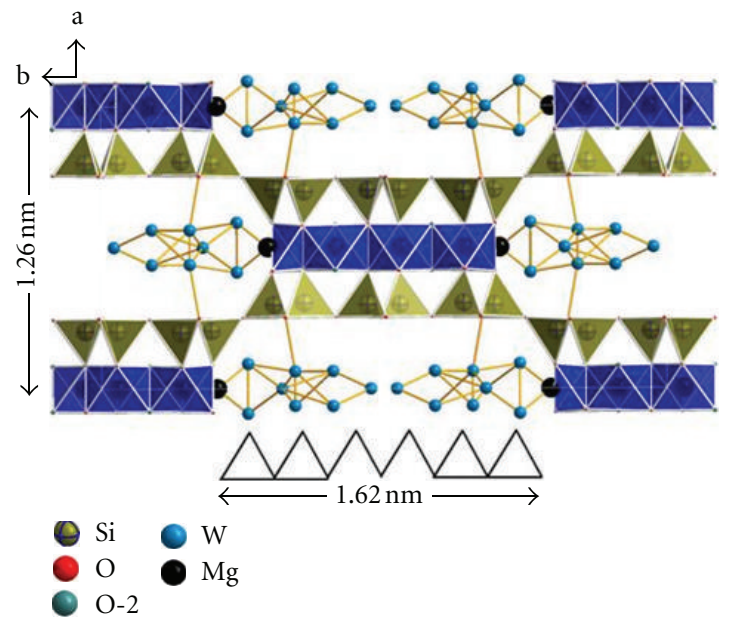

(b)

FIGURE 8: Diagram of crystal structure of sepiolite ((a) along $a$-axis direction; (b) along $c$-axis direction).

of the channel is only $0.63 \mathrm{~nm}$ after decreasing the space occupied by silica tetrahedron net surfaces and magnesium oxygen octahedron core layer. The length of the channel is $0.63 \times 1.60 \mathrm{~nm}$ in condition of containing eight zeolite water, which cannot be measured quantitatively by BET method [13-15].

\section{Conclusions}

TEM observation of sepiolite fiber and section sample shows that sepiolite crystal not only contains a lot of structural micropores parallel to the fiber axis, but it also contains a large amount of short-range radial micropores perpendicular to the fiber axis. Radial micropores connect the micropores parallel to the fiber axis to each other, forming the threedimensional internally connected channel network structure. The diameter of the micropores is mainly from 2 to $5 \mathrm{~nm}$, in well accordance with the results of nitrogen adsorption BET analysis. TEM observation and XRD analysis prove that sepiolite crystal contains structural micropores parallel to the $c$-axis, but the real size and volume of these micropores cannot be detected quantitatively so far, and the formation mechanism of many microporous channels perpendicular to the $c$-axis in crystal needs further discussion.

\section{Acknowledgments}

This research was financially supported by the Application Foundation and Advanced Technology Research Program of Tianjin city (Grant no. 12JCQNJC02100), Application Foundation and Advanced Technology Research Program of Tianjin city (Grant no. 10JCZDJC22300), and Natural Science Foundation of Hebei province (Grant no. E2009000069).

\section{References}

[1] B. S. Nagy and W. F. Bradley, "The structural scheme of sepiolite," America Mineralogist, vol. 40, pp. 885-892, 1955.
[2] K. Brauner and A. Preisinger, "Struktur und entstehung des sepioliths," Tschermaks Mineralogische und Petrographische Mitteilungen, vol. 6, no. 1-2, pp. 120-140, 1956.

[3] M. P. S. Krekeler and S. Guggenheim, "Defects in microstructure in palygorskite-sepiolite minerals: a transmission electron microscopy (TEM) study," Applied Clay Science, vol. 39, no. 12, pp. 98-105, 2008.

[4] A. Miura, K. Nakazawa, T. Takei et al., "Acid-, base-, and heatinduced degradation behavior of Chinese sepiolite," Ceramics International, vol. 38, pp. 4677-4684, 2012.

[5] S. Tunç, O. Duman, and A. Çetinkaya, "Electrokinetic and rheological properties of sepiolite suspensions in the presence of hexadecyltrimethylammonium bromide," Colloids and Surfaces A, vol. 377, no. 1-3, pp. 123-129, 2011.

[6] H. X. Chen, H. Z. Lu, Y. Zhou, M. S. Zheng, C. M. Ke, and D. L. Zeng, "Study on thermal properties of polyurethane nanocomposites based on organo-sepiolite," Polymer Degradation and Stability, vol. 97, pp. 242-247, 2012.

[7] E. Işçi and S. I. Turutoğlu, "Stabilization of the mixture of bentonite and sepiolite as a water based drilling fluid," Journal of Petroleum Science and Engineering, vol. 76, no. 1-2, pp. 1-5, 2011.

[8] N. Volle, F. Giulieri, A. Burr, S. Pagnotta, and A. M. Chaze, "Controlled interactions between silanol groups at the surface of sepiolite and an acrylate matrix: consequences on the thermal and mechanical properties," Materials Chemistry and Physics, vol. 134, pp. 417-424, 2012.

[9] A. S. Ozcan and O. Gok, "Structural characterization of dodecyltrimethylammonium (DTMA) bromide modified sepiolite and its adsorption isotherm studies," Journal of Molecular Structure, vol. 1007, pp. 36-44, 2012.

[10] M. Suarez and E. Garcia-Romero, "Variability of the surface properties of sepiolite," Applied Clay Science, vol. 67-68, pp. 72-82, 2012.

[11] Q. W. Zhu, Y. H. Zhang, F. Z. Lv, P. K. Chu, Z. F. Ye, and F. S. Zhou, "Cuprous oxide created on sepiolite: preparation, characterization, and photocatalytic activity in treatment of red water from 2,4,6-trinitrotoluene manufacturing," Journal of Hazardous Materials, vol. 217-218, pp. 11-18, 2012.

[12] K. M. Fang, L. Zheng, K. Fang, and L. Huang, "Revealing microstructure of fine particles by TEM," China Particuology, vol. 1, pp. 88-90, 2003. 
[13] S. Inagaki, Y. Fukushima, H. Doi, and O. Kamigaito, "Pore size distribution and adsorption selectivity of sepiolite," Clay Minerals, vol. 25, no. 1, pp. 99-105, 1990.

[14] A. A. Goktas, Z. Misirli, and T. Baykara, "Sintering behaviour of sepiolite," Ceramics International, vol. 23, no. 4, pp. 305311, 1997.

[15] H. Toshiyuki, T. Atsumu, Y. Atsushi, and O. Ryohei, "Model calculation of sepiolite surface areas," Clays and Clay Minerals, vol. 43, pp. 391-396, 1995. 

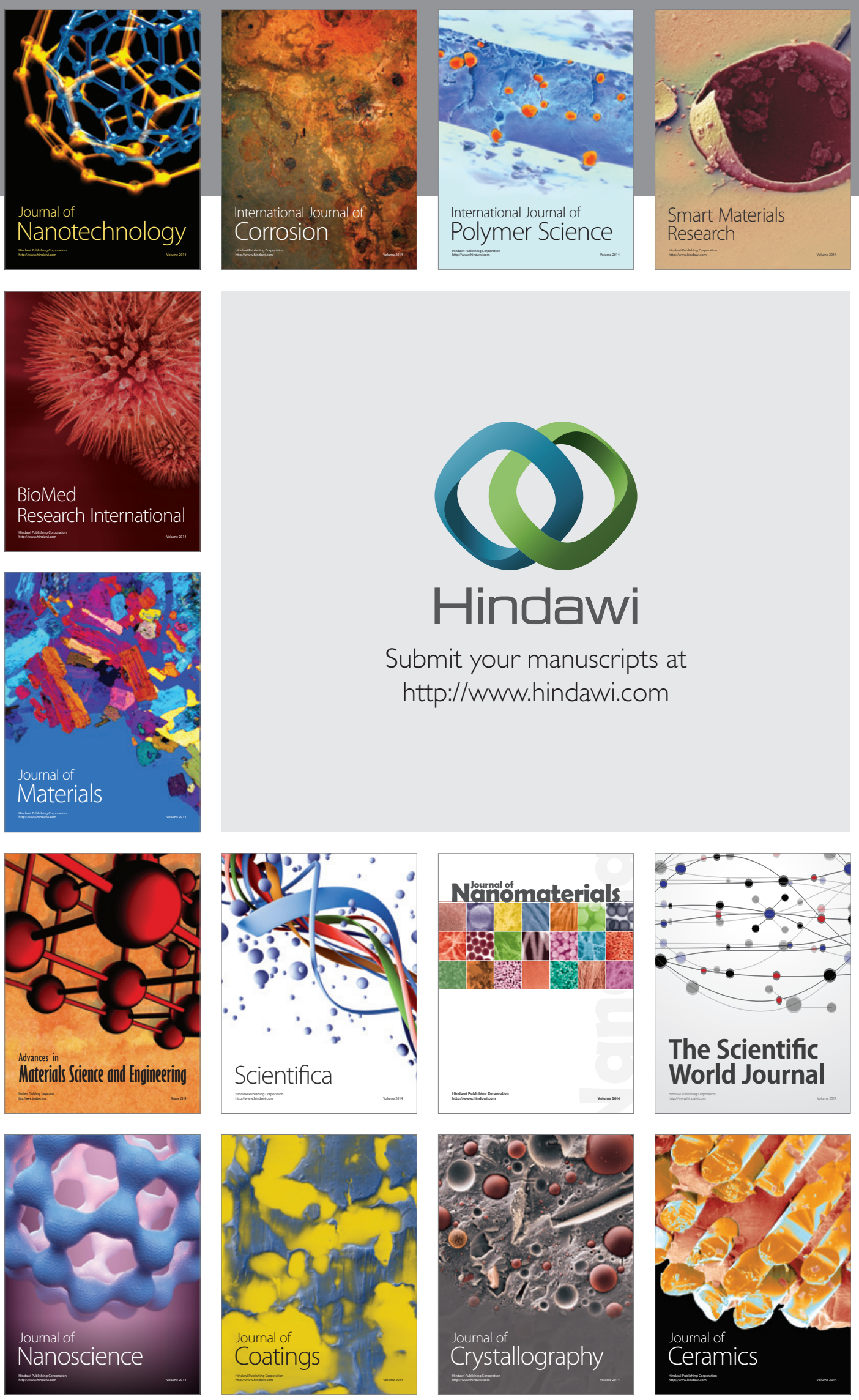

The Scientific World Journal

Submit your manuscripts at

http://www.hindawi.com

\section{World Journal}

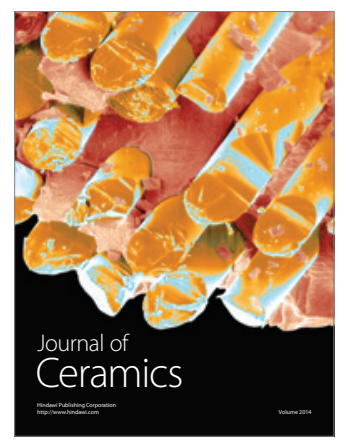

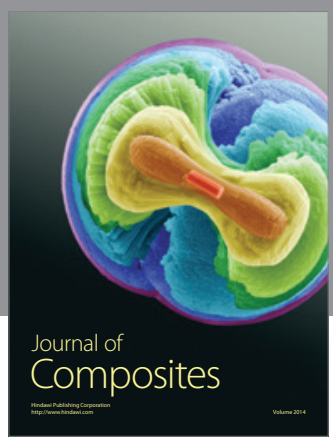
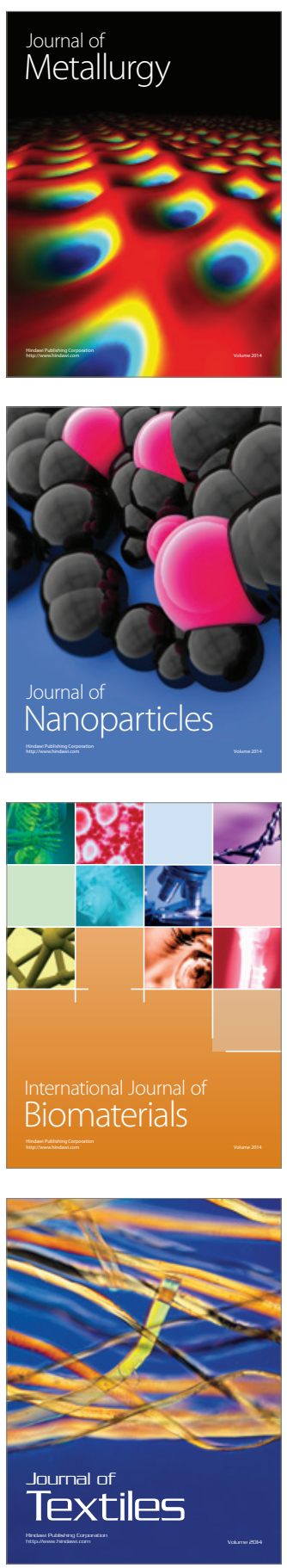